\title{
Trilhas afetam comunidades arbóreas florestais? Dois levantamentos na Floresta Atlântica do sudeste brasileiro ${ }^{1}$
}

\author{
Pedro Vasconcellos Eisenlohr ${ }^{2,5}$, Maria Margarida da Rocha Fiuza de Melo ${ }^{3}$ e Adriano Valentin da Silva ${ }^{4}$
}

Recebido: 30.10.2008; aceito: 23.04.2009

\begin{abstract}
Do trails affect forest tree communities? Two surveys in the Atlantic Forest of southeastern Brazil). Forest trails can influence the composition and structure of plant communities by causing abiotic changes along their course and in the vicinity. To understand the behavior of the floristic and structural vegetation along the trails, two surveys were conducted in the Atlantic Forest of southeastern Brazil, in the "Parque Estadual das Fontes do Ipiranga", São Paulo, State of São Paulo, and in the "Mata da Biologia", Viçosa, State of Minas Gerais, using identical minimum diameter at breast height and sample areas. For the data analysis, ANOVA and chi-square test were chosen. There were variations in density and number of upright dead plants in both surveys, with higher values alongside the trail. Both fragments showed no relationship between distribution of plants per dispersion syndrome and distance from the trail. It was found that the trails interfere with tree vegetation in both fragments, and it is suggested that trails should come to be treated as a potential source of impact in forest restoration programs.
\end{abstract}

Key words: conservation, edge effect, phytosociology

RESUMO - (Trilhas afetam comunidades arbóreas florestais? Dois levantamentos na Floresta Atlântica do sudeste brasileiro). As trilhas podem influenciar a composição e a estrutura de comunidades vegetais, por causar modificações abióticas em seu local de ocorrência e proximidades. Buscando compreender o comportamento florístico e estrutural da vegetação em relação a trilhas, foram realizados dois levantamentos na Floresta Atlântica do sudeste brasileiro, no Parque Estadual das Fontes do Ipiranga, São Paulo, SP, e na Mata da Biologia, Viçosa, MG, utilizando critérios de inclusão e áreas amostrais idênticos. $\mathrm{Na}$ análise de dados, optou-se pela ANOVA e teste qui-quadrado. Houve variação na densidade e no número de indivíduos mortos em pé em ambos os levantamentos, com maiores valores na borda da trilha. Ambos os fragmentos não apresentaram relação entre distribuição de indivíduos por síndrome de dispersão e distância da trilha. Verificou-se que as trilhas exercem interferência sobre a vegetação arbórea em ambos os fragmentos e, assim, sugere-se que programas de restauração florestal passem a considerar as trilhas como fonte potencial de impacto.

Palavras-chave: conservação, efeito de borda, fitossociologia

\section{Introdução}

A busca por áreas naturais para recreação tem se intensificado devido à vida agitada nos centros urbanos (Ladeira et al. 2007), contribuindo para um substancial aumento no volume de turistas que visitam áreas protegidas almejando descanso e fuga da rotina (IUCN 1993 apud Robim et al. 2004). O contato com a natureza constitui uma das maiores motivações das viagens de lazer (Ladeira et al.2007), possibilitando inclusive a realização de atividades didáticas na área de Educação Ambiental, por meio de trilhas interpretativas (Oliveira et al. 1999).

Uma das formas de recreação que colocam o homem em contato com a natureza é o ecoturismo. Segundo a definição adotada pela The International Union for Conservation of Nature and Natural Resources (IUCN), ecoturismo consiste em atividades de visitação da população a áreas naturais a fim de desfrutar e apreciar a natureza (e qualquer característica

1. Parte da Dissertação de Mestrado do primeiro autor pelo Programa de Pós-Graduação em Biodiversidade Vegetal e Meio Ambiente do Instituto de Botânica, São Paulo, SP, Brasil.

2. Universidade Estadual de Campinas, Programa de Pós-Graduação em Biologia Vegetal, Caixa Postal 6109, 13083-970 Campinas, SP, Brasil

3. Instituto de Botânica, Seção de Curadoria do Herbário, Avenida Miguel Estéfano, 3687, 04301-902 São Paulo, SP, Brasil

4. Universidade Federal de Viçosa, Departamento de Biologia Vegetal, Avenida PH Rolfs s/n, 36570-000 Viçosa, MG, Brasil

5. Autor para correspondência: pedrov.eisenlohr@gmail.com 
cultural nelas existente, tanto passada quanto presente), que promova a conservação; deve, ainda, estar atrelado a um baixo nível de degradação sobre o meio ambiente e promover, de maneira benéfica, o envolvimento sócio-econômico ativo das populações locais (Drumm $\&$ Moore 2002). Dessa forma, o ecoturismo pode ser visto como um paradoxo, pois ao mesmo tempo em que explora hábitats naturais, também depende da preservação desses (Kent 2003).

Muitas das visitas ecoturísticas desenvolvem-se em Unidades de Conservação nas áreas destinadas ao uso público, originalmente planejadas para atender a uma demanda menor do que a atual, com atividades pouco danosas e com um público muito diferente daquele que atualmente frequenta essas áreas naturais protegidas (Freixêdas-Vieira et al. 2000). Aliado a isso, as trilhas que atravessam ou circunscrevem-se às Unidades de Conservação passam normalmente por ambientes naturais muitas vezes frágeis e carentes de proteção (Andrade 2003).

Para Pires et al. (2004a), os efeitos das atividades humanas nos ecossistemas sãoos principais responsáveis para que uma biorregião seja considerada sustentável ou não. A integridade ecológica dos ecossistemas naturais implica, necessariamente, na manutenção de suas áreas naturais em condições satisfatórias de qualidade ambiental, de forma a garantir a continuidade dos processos ecológicos ao longo do tempo (Pires et al. 2004b). Nesse contexto, para conciliar o uso recreativo em Unidades de Conservação com seus objetivos primários (conservação dos recursos naturais e pesquisa científica), os locais designados para o desenvolvimento de atividades de uso público devem ser manejados para controlar os efeitos negativos sobre o ambiente e garantir a qualidade de experiência do visitante (Freixêdas-Vieira et al. 2000).

O problema é que muitas vezes esses cuidados não são respeitados. O uso irrestrito de recreação em áreas naturais tende a prejudicar as comunidades vegetais (Cole 1978) e esse efeito tende a ser sentido a partir da abertura e da utilização das trilhas. Os impactos das trilhas sobre a vegetação podem ser diretos, por dano mecânico causado pela presença antrópica, ou indiretos, por mudanças causadas nas propriedades físicas e químicas do solo (Roncero-Siles 2003). Também em relação aos solos, Roovers et al. (2004) alertaram para os efeitos da visitação intensiva sobre a sua compactação excessiva e sobre os efeitos desta alteração sobre a cobertura e a composição florística de uma determinada área de floresta, ressaltando que outros fatores bióticos e abióticos possam vir a ser fortemente alterados como resultado desse processo. Esses impactos podem afetar a capacidade reprodutiva das espécies vegetais por interferir em processos como crescimento, germinação e estabelecimento de sementes (Cole 1978, Roovers et al. 2004).

A intensidade do efeito causado pela trilha varia, dependendo da vulnerabilidade da vegetação afetada, da frequência de uso da trilha e do regime de manutenção da mesma (Cole 1978, Roovers et al. 2004). Estudos abordando a susceptibilidade de diferentes formações vegetais às mudanças resultantes da construção e uso de trilhas são importantes para que se disponha de mais informações a fim de conceber sistemas de trilhas que evitem regiões mais frágeis (Cole 1978).

Devido à escassez de estudos em áreas florestais atravessadas por trilhas, não se sabe ainda se o efeito resultante poderia alterar aspectos estruturais e estratégias de dispersão nas florestas. Sabe-se que as bordas oriundas da fragmentação de ecossistemas naturais modificam as condições ambientais, podendo elevar a mortalidade de árvores e favorecer o estabelecimento de espécies que não sejam daquele ecossistema (Antunes et al. 2000, Rodrigues \& Nascimento 2006). Espécies anemocóricas poderiam, também, ser favorecidas em tais condições, devido à maior exposição aos ventos do que no interior da floresta (Nunes et al. 2003). Esses efeitos também poderiam ser causados por trilhas? Os estudos de Cole (1978) e Roovers et al. (2004) indicam que sim, porém isso provavelmente se daria em menor magnitude em relação a bordas de fragmentos florestais, devido à menor dimensão das trilhas e também à menor exposição a fatores abióticos (vento e radiação solar, principalmente), visto que as trilhas em geral encontram-se no interior das florestas. O grande problema parece ser testar, com controle mínimo das variáveis envolvidas, essa diferença entre efeito de borda e efeito de trilha, não sendo este um objetivo do presente trabalho.

O objetivo aqui foi avaliar a interferência causada pela abertura e utilização de trilhas em dois fragmentos de Floresta Atlântica no sudeste brasileiro, integrando dados de dois levantamentos (Hirata 2006 e o efetuado na Mata da Biologia, Viçosa, MG, aqui apresentado) realizados com esse enfoque. A hipótese testada foi que as trilhas influenciam a estrutura do componente arbóreo nas florestas investigadas, por variação nas tabelas fitossociológicas e nos parâmetros densidade, mortalidade e distribuição dos indivíduos por síndromes de dispersão. 


\section{Material e métodos}

Áreas de estudo - O Parque Estadual das Fontes do Ipiranga (PEFI) localiza-se a aproximadamente $23^{\circ} 38^{\prime} \mathrm{S}$ e $46^{\circ} 38^{\prime} \mathrm{W}$, no Planalto Paulistano, no extremo sul da cidade de São Paulo, SP. Em altitude entre 770 e $825 \mathrm{~m}$, sua Reserva Biológica abrange 357 ha e configura-se como área de transição entre as Florestas Ombrófila Densa e Estacional Semidecídua (Veloso et al. 1991) da Mata Atlântica sensu lato (Oliveira Filho \& Fontes 2000). Segundo o sistema de Koeppen, o clima da região classifica-se como Cwb. De acordo com Gomes \& Mantovani (2001), o principal tipo de solo na região é o Latossolo Vermelho-Amarelo.

A Mata da Biologia localiza-se no Campus da Universidade Federal de Viçosa, a aproximadamente $20^{\circ} 45^{\prime} \mathrm{S}$ e $42^{\circ} 51^{\prime} \mathrm{W}$, no município de Viçosa, Zona da Mata de Minas Gerais. Possui 75 ha, sendo classificada como Floresta Estacional Semidecídua (Veloso et al. 1991), e situa-se a $650 \mathrm{~m}$ de altitude média. O clima da região é do tipo Cwa, segundo o sistema de Koeppen, com déficit hídrico entre abril e setembro. Predominam, em seus solos, as seguintes classes: Latossolo Amarelo, nos topos convexos; Latossolo Vermelho-Amarelo, nas encostas das elevações; Podzólico Vermelho-Amarelo Câmbico, nos terraços; Solos Aluviais associados aos Hidromórficos, nos fundos de vales (Ferreira Júnior et al. 2007).

Amostragem da vegetação - Foi utilizado o método de parcelas disjuntas (Mueller-Dombois \& Ellenberg 1974) de $100 \mathrm{~m}^{2}$ cada, sendo a amostragem do tipo sistemática. No PEFI, foram instaladas 10 parcelas a $7 \mathrm{~m}$ de distância de cada uma das trilhas. As 10 parcelas da trilha de maior largura e utilização foram convencionalmente denominadas "Setor Terra Batida", e as 10 em trilha de menor largura e utilização, "Setor Fontes do Ipiranga". Como área controle, 10 parcelas foram estudadas no interior da floresta ("Setor Controle"). As planilhas de campo obtidas por Hirata (2006) foram utilizadas como base para as análises relativas ao PEFI.

Na Mata da Biologia, optou-se por instalar 10 parcelas na área central de uma trilha de uso intermediário, sendo cinco na borda (a $1 \mathrm{~m}$ da trilha, recebendo a denominação de "Setor Borda") e outras cinco a 7 m da trilha ("Setor Distância Média"). Não foram alocadas mais parcelas nesses setores devido às fortes variações de topografia e solos encontradas (Ferreira Júnior et al. 2007 e observações de campo), o que poderia mascarar severamente os resultados, tendo em vista que o efeito investigado é presumivelmente muito específico. Como área controle, foram alocadas
20 parcelas no interior da mata, sendo $10 \mathrm{em}$ semelhantes condições de solo e topografia ("Setor Controle") e outras 10 em condições variadas de solo e topografia ("Setor Floresta"). Esse desenho amostral foi delineado de maneira a caracterizar com propriedade e confiabilidade a vegetação sem influência da trilha em um fragmento onde, conforme dito acima, descritores ambientais como solos e topografia sofrem modificações abruptas em áreas próximas. Assim, a área total amostrada em cada floresta foi de 0,3 ha.

Em ambos os levantamentos, os setores de interior de mata situavam-se a pelo menos $50 \mathrm{~m}$ de qualquer fonte de perturbação antrópica, e os setores próximos às trilhas encontravam-se distantes (pelo menos 200 m) da borda do fragmento, de forma que um possível efeito agressivo, que não o investigado, pudesse ser minimizado ou mesmo suprimido.

No interior das parcelas, todos os indivíduos, vivos ou mortos em pé, com diâmetro do caule a $1,30 \mathrm{~m}$ de altura do solo (DAP) igual ou superior a $2,5 \mathrm{~cm}$ foram amostrados. As coletas dos ramos para identificação taxonômica seguiram o método sugerido por Fidalgo \& Bononi (1989). A nomenclatura dos binômios seguiu o W3-Tropicos do MOBOT (2007) e as famílias foram classificadas segundo a proposta da APG II (2003).

Processamento dos dados - As planilhas contendo espécies, famílias, indivíduos e seus respectivos DAP foram processadas no software MATA NATIVA 2.0 (CIENTEC 2004), sendo obtidos os parâmetros estimados de Frequência, Densidade e Dominância (relativas e absolutas) e Índice do Valor de Importância (IVI). Esses parâmetros são descritos e suas fórmulas apresentadas em Mueller-Dombois \& Ellenberg (1974) e Martins (1991). Foram, ainda, calculados os índices de diversidade de Shannon (H') e de equabilidade de Pielou (J'), cujas fórmulas e explicações são dadas por Magurran (2004).

Classificação das espécies - Cada espécie foi classificada quanto à síndrome de dispersão (zoocórica, anemocórica ou autocórica) segundo Pijl (1982), por meio de consulta à ampla literatura. Procurou-se, a partir dessas informações, comparar as distribuições de indivíduos, por síndrome, em cada um dos setores, em cada fragmento.

Análises estatísticas - A fim de se inferir se houve diferença significativa entre as médias de densidade total e de indivíduos mortos em pé, foi aplicada ANOVA e, quando necessário, teste a posteriori de Tukey-Kramer (Zar 1999), com $\alpha=5 \%$. A hipótese de nulidade previa ausência de variação entre as 
médias desses parâmetros nos diferentes níveis de interferência (setores) causada pela trilha. Para conferir a normalidade dos dados, foi empregado o teste de Kolmogorov-Smirnov (Zar 1999).

Com relação à distribuição de indivíduos por síndromes de dispersão entre os diferentes setores amostrados, foi aplicado o teste qui-quadrado de comparação entre proporções (Callegari-Jacques 2003), com $\alpha=5 \%$. Modernas teorias estatísticas têm advogado a favor de restrições menos rígidas ao uso desse teste (Zar 1999) e, com base nessa abordagem, foi possível utilizar os baixos valores de autocoria na presente análise. A hipótese testada foi que, em cada floresta (PEFI/Mata da Biologia), a proporção de indivíduos anemocóricos, zoocóricos e autocóricos seria a mesma em todos os setores. Em Viçosa, de modo a manter o mesmo $\mathrm{N}$ amostral (10) em todos os níveis categóricos (Trilha/Controle/Floresta), optou-se (apenas para o teste qui-quadrado) por fundir os setores Borda e Distância Média em apenas um (denominado Trilha), por ambos estarem, em comparação com os setores de interior de mata, próximos à trilha. Todos os cálculos foram executados segundo Zar (1999) e Callegari-Jacques (2003).

As listagens florísticas aqui obtidas não foram comparadas com outras disponíveis para as respectivas regiões em razão dos objetivos do presente trabalho serem muito específicos e o DAP mínimo de inclusão ser diferente daqueles habitualmente utilizados em fitossociologia.

\section{Resultados e Discussão}

No estudo fitossociológico realizado no Parque Estadual das Fontes do Ipiranga (PEFI), foram amostrados 802 indivíduos distribuídos em 116 espécies, incluindo a categoria "Mortas", e em 36 famílias (Hirata 2006). Esse levantamento apresentou H' de 4,25 e J' de 0,89 . Nos setores, os H' foram de 3,68 (Terra Batida), 3,74 (Fontes do Ipiranga) e 3,83 (Controle). Já os valores de J' foram de 0,88, 0,93 e 0,93 , respectivamente.

As famílias mais ricas foram Lauraceae, com 15 espécies, Fabaceae e Myrtaceae (14 cada), Rubiaceae (nove) e Arecaceae e Euphorbiaceae (seis cada). Essas famílias são usualmente apontadas na literatura como bem representadas em Florestas Montanas ou Submontanas da costa atlântica dos estados de São Paulo e Rio de Janeiro (Tabarelli \& Mantovani 1999, Catharino et al. 2006).

Houve diferenciação notável na estrutura da comunidade arbórea conforme o nível de interferência das trilhas (tabela 1). No setor Terra Batida predominou Archontophoenix cunninghamiana; no setor Trilha Fontes do Ipiranga, a categoria das Mortas, e no setor Controle, Cupania emarginata. Cabe ressaltar que a espécie exótica Archontophoenix cunninghamiana ocupou a segunda posição em IVI na amostragem total e, segundo Hirata (2006), essa espécie pode estar sendo favorecida pelas condições proporcionadas pelas trilhas. Dislish et al. (2002), em estudo sobre a dinâmica da ocupação espacial de A. cunninghamiana, identificou-a como a espécie invasora mais importante em um fragmento florestal com características semelhantes às do PEFI.

Na Mata da Biologia, Viçosa, MG, foram amostrados 1.104 indivíduos pertencentes a 130 espécies, que se apresentaram distribuídas em 34 famílias somadas às categorias "Indeterminadas" e "Mortas". O Índice de Diversidade de Shannon (H') foi de 3,69 e o Índice de Equabilidade de Pielou (J'), de 0,76 . Nos setores amostrados, os H' foram de 3,22 (Borda), 3,17 (Distância Média), 3,39 (Controle) e 3,40 (Floresta). Os J', nesses mesmos setores, foram de $0,79,0,79,0,82$ e 0,81 , respectivamente.

A família com maior riqueza foi Fabaceae, com 21 espécies, seguida de Lauraceae e Rubiaceae (14 cada), Annonaceae (oito), Meliaceae (sete) e Myrtaceae (seis). A predominância das três primeiras, especialmente Fabaceae, já havia sido documentada em levantamentos realizados na região por vários autores, dentre os quais Meira-Neto \& Martins (2002) e Marangon et al. (2003).

Em termos de estrutura da comunidade arbórea, duas espécies merecem comentários por seu destaque nos parâmetros analisados. Chrysophyllum flexuosum esteve presente em todas as parcelas dos setores Borda e Distância Média, e o fez com elevada densidade de indivíduos, o que concorreu diretamente para que seu IVI fosse o maior entre as espécies amostradas nesses setores (tabela 2). Por sua vez, no interior da floresta quem se destacou foi a exótica Coffea arabica, presente em $90 \%$ das parcelas daquela área, com 65 indivíduos no setor Controle e 76 no setor Floresta; esse fato certamente está associado com o histórico da Mata da Biologia, que antes de ser incorporada à Universidade Federal de Viçosa era um grande cafezal. A presença dessa espécie, porém, é considerada negativa tanto para os representantes dos estratos herbáceo e arbustivo como para a regeneração das espécies arbóreas dos demais estratos da floresta, podendo inclusive causar inibição química de germinação e crescimento de espécies sensíveis (Lopes et al. 2002). Caberia, em um estudo futuro, investigar a reduzida participação do café na região próxima à trilha. 
Tabela 1. Estimativa dos parâmetros fitossociológicos para a amostragem realizada por Hirata (2006) no Parque Estadual das Fontes do Ipiranga, São Paulo, SP, Brasil. N = número de indivíduos; DA = densidade absoluta; DR = densidade relativa; FA = freqüência absoluta; $\mathrm{FR}=$ frequiência relativa; DoA = dominância absoluta; DoR = dominância relativa; IVI = índice do valor de importância $(\%)$. São apresentadas apenas as 10 espécies de mais alto IVI para cada setor.

Table 1. Estimation of the phytosociological parameters for the sampling conducted in the Parque Estadual Fontes do Ipiranga, São Paulo, SP, Brazil (Hirata 2006). $\mathrm{N}=$ number of individuals; DA = absolute density; DR = relative density; FA = absolute frequency; FR = relative frequency; DoA = absolute dominance; DoR = relative dominance; IVI = importance value index $(\%)$. Only the 10 higher species in IVI for each sector are presented.

\begin{tabular}{|c|c|c|c|c|c|c|c|c|}
\hline Espécie & $\mathrm{N}$ & DA & DR & FA & FR & DoA & DoR & IVI \\
\hline \multicolumn{9}{|l|}{ TERRA BATIDA } \\
\hline Archontophoenix cunninghamiana & 26 & 260 & 11,61 & 80 & 5,71 & 5,12 & 11,66 & 9,66 \\
\hline Syagrus romanzoffiana & 16 & 160 & 7,14 & 60 & 4,29 & 3,40 & 7,74 & 6,39 \\
\hline Alchornea triplinervia & 3 & 30 & 1,34 & 30 & 2,14 & 5,58 & 12,70 & 5,39 \\
\hline Mortas & 13 & 130 & 5,80 & 70 & 5,00 & 2,19 & 4,98 & 5,26 \\
\hline Eugenia excelsa & 8 & 80 & 3,57 & 60 & 4,29 & 3,16 & 7,20 & 5,02 \\
\hline Myrcia floribunda & 8 & 80 & 3,57 & 60 & 4,29 & 2,21 & 5,03 & 4,30 \\
\hline Guarea macrophylla & 9 & 90 & 4,02 & 60 & 4,29 & 1,45 & 3,30 & 3,87 \\
\hline Jacaratia heptaphylla & 10 & 100 & 4,46 & 50 & 3,57 & 1,34 & 3,06 & 3,70 \\
\hline Euterpe edulis & 12 & 120 & 5,36 & 30 & 2,14 & 1,42 & 3,23 & 3,58 \\
\hline Cedrela fissilis & 7 & 70 & 3,13 & 20 & 1,43 & 2,42 & 5,50 & 3,35 \\
\hline \multicolumn{9}{|l|}{ FONTES DO IPIRANGA } \\
\hline Mortas & 27 & 270 & 8,41 & 90 & 4,62 & 3,06 & 10,90 & 7,97 \\
\hline Psychotria cephalantha & 8 & 80 & 2,49 & 60 & 3,08 & 3,34 & 11,88 & 5,82 \\
\hline Euterpe edulis & 20 & 200 & 6,23 & 100 & 5,13 & 1,29 & 4,60 & 5,32 \\
\hline Amaioua guianensis & 9 & 90 & 2,80 & 60 & 3,08 & 1,69 & 6,02 & 3,97 \\
\hline Syagrus romanzoffiana & 15 & 150 & 4,67 & 50 & 2,56 & 0,95 & 3,39 & 3,54 \\
\hline Calyptranthes grandifolia & 5 & 50 & 1,56 & 30 & 1,54 & 2,07 & 7,36 & 3,49 \\
\hline Archontophoenix cunninghamiana & 18 & 180 & 5,61 & 30 & 1,54 & 0,91 & 3,24 & 3,46 \\
\hline Lacistema lucidum & 8 & 80 & 2,49 & 60 & 3,08 & 1,30 & 4,63 & 3,40 \\
\hline Cyathea delgadii & 11 & 110 & 3,43 & 50 & 2,56 & 1,07 & 3,79 & 3,26 \\
\hline Eugenia excelsa & 7 & 70 & 2,18 & 60 & 3,08 & 1,15 & 4,08 & 3,11 \\
\hline \multicolumn{9}{|l|}{ CONTROLE } \\
\hline Cupania emarginata & 5 & 50 & 1,95 & 40 & 2,33 & 8,68 & 22,46 & 8,91 \\
\hline Posoqueria acutifólia & 10 & 100 & 3,89 & 70 & 4,07 & 4,15 & 10,74 & 6,23 \\
\hline Actinostemon klotzskii & 22 & 220 & 8,56 & 60 & 3,49 & 1,97 & 5,09 & 5,71 \\
\hline Mortas & 10 & 100 & 3,89 & 50 & 2,91 & 1,68 & 4,36 & 3,72 \\
\hline Maytenus robusta & 5 & 50 & 1,95 & 50 & 2,91 & 2,18 & 5,65 & 3,50 \\
\hline Euterpe edulis & 9 & 90 & 3,50 & 40 & 2,33 & 1,80 & 4,65 & 3,49 \\
\hline Nectandra megapotamica & 2 & 20 & 0,78 & 20 & 1,16 & 2,79 & 7,22 & 3,05 \\
\hline Syagrus romanzoffiana & 9 & 90 & 3,50 & 40 & 2,33 & 1,23 & 3,18 & 3,00 \\
\hline Sorocea bonplandii & 13 & 130 & 5,06 & 50 & 2,91 & 0,28 & 0,71 & 2,89 \\
\hline Campomanesia guaviroba & 3 & 30 & 1,17 & 30 & 1,74 & 2,00 & 5,17 & 2,69 \\
\hline
\end{tabular}


Tabela 2. Estimativa dos parâmetros fitossociológicos para a amostragem realizada na Mata da Biologia, Viçosa, MG, Brasil. N = número de indivíduos; $\mathrm{DA}=$ densidade absoluta; $\mathrm{DR}=$ densidade relativa; $\mathrm{FA}=$ freqüência absoluta; $\mathrm{FR}=$ freqüência relativa; DoA = dominância absoluta; DoR = dominância relativa; IVI = índice do valor de importância (\%). São apresentadas apenas as 10 espécies de mais alto IVI para cada setor.

Table 2. Estimation of the phytosociological parameters for the sampling conducted in the Mata da Biologia, Viçosa, MG, Brazil. $\mathrm{N}=$ number of individuals; DA = absolute density; DR = relative density; FA = absolute frequency; FR = relative frequency; DoA = absolute dominance; DoR = relative dominance; IVI = importance value index $(\%)$. Only the 10 higher species in IVI for each sector are presented.

\begin{tabular}{|c|c|c|c|c|c|c|c|c|}
\hline Espécie & $\mathrm{N}$ & DA & DR & FA & FR & DoA & DoR & IVI \\
\hline \multicolumn{9}{|l|}{ BORDA } \\
\hline Chrysophyllum flexuosum & 56 & 1120 & 22,40 & 100 & 4,76 & 5,52 & 9,97 & 12,38 \\
\hline Nectandra lanceolata & 9 & 180 & 3,60 & 80 & 3,81 & 9,44 & 17,05 & 8,15 \\
\hline Mortas & 22 & 440 & 8,80 & 100 & 4,76 & 4,60 & 8,32 & 7,29 \\
\hline Sorocea bonplandii & 23 & 460 & 9,20 & 80 & 3,81 & 0,94 & 1,69 & 4,90 \\
\hline Trichilia lepidota & 10 & 200 & 4,00 & 60 & 2,86 & 3,16 & 5,71 & 4,19 \\
\hline Alchornea glandulosa & 5 & 100 & 2,00 & 60 & 2,86 & 4,24 & 7,67 & 4,18 \\
\hline Anadenanthera peregrina & 4 & 80 & 1,60 & 60 & 2,86 & 4,08 & 7,38 & 3,94 \\
\hline Trichilia pallida & 13 & 260 & 5,20 & 100 & 4,76 & 0,81 & 1,47 & 3,81 \\
\hline Prunus sellowii & 12 & 240 & 4,80 & 60 & 2,86 & 1,68 & 3,04 & 3,57 \\
\hline Xylosma prockia & 9 & 180 & 3,60 & 60 & 2,86 & 1,69 & 3,06 & 3,17 \\
\hline \multicolumn{9}{|l|}{ DISTÂNCIA MÉDIA } \\
\hline Chrysophyllum flexuosum & 53 & 1060 & 29,28 & 100 & 5,26 & 5,56 & 14,93 & 16,49 \\
\hline Piptadenia gonoacantha & 3 & 60 & 1,66 & 40 & 2,11 & 7,41 & 19,90 & 7,89 \\
\hline Trichilia lepidota & 7 & 140 & 3,87 & 80 & 4,21 & 2,42 & 6,50 & 4,86 \\
\hline Machaerium stipitatum & 5 & 100 & 2,76 & 60 & 3,16 & 3,20 & 8,58 & 4,83 \\
\hline Nectandra lanceolata & 3 & 60 & 1,66 & 60 & 3,16 & 3,12 & 8,37 & 4,39 \\
\hline Mortas & 10 & 200 & 5,52 & 80 & 4,21 & 1,06 & 2,86 & 4,20 \\
\hline Copaifera sp. & 1 & 20 & 0,55 & 20 & 1,05 & 3,25 & 8,71 & 3,44 \\
\hline Xylosma prockia & 7 & 140 & 3,87 & 80 & 4,21 & 0,80 & 2,13 & 3,40 \\
\hline Sorocea bonplandii & 6 & 120 & 3,31 & 60 & 3,16 & 0,64 & 1,71 & 2,73 \\
\hline Protium warmingianum & 6 & 120 & 3,31 & 60 & 3,16 & 0,62 & 1,66 & 2,71 \\
\hline \multicolumn{9}{|l|}{ CONTROLE } \\
\hline Coffea arabica & 65 & 650 & 19,70 & 90 & 5,33 & 0,63 & 2,42 & 9,15 \\
\hline Nectandra lanceolata & 10 & 100 & 3,03 & 60 & 3,55 & 3,50 & 13,34 & 6,64 \\
\hline Prunus sellowii & 22 & 220 & 6,67 & 90 & 5,33 & 1,64 & 6,27 & 6,09 \\
\hline Allophylus edulis & 6 & 60 & 1,82 & 40 & 2,37 & 3,55 & 13,53 & 5,90 \\
\hline Trichilia lepidota & 14 & 140 & 4,24 & 60 & 3,55 & 1,62 & 6,18 & 4,66 \\
\hline Trichilia pallida & 18 & 180 & 5,45 & 80 & 4,73 & 0,72 & 2,75 & 4,31 \\
\hline Chrysophyllum flexuosum & 18 & 180 & 5,45 & 50 & 2,96 & 0,53 & 2,02 & 3,48 \\
\hline Cabralea canjerana & 8 & 80 & 2,42 & 60 & 3,55 & 1,02 & 3,88 & 3,28 \\
\hline Piptadenia gonoacantha & 6 & 60 & 1,82 & 50 & 2,96 & 1,31 & 4,99 & 3,26 \\
\hline Citronella megaphylla & 16 & 160 & 4,85 & 50 & 2,96 & 0,43 & 1,63 & 3,15 \\
\hline \multicolumn{9}{|l|}{ FLORESTA } \\
\hline Coffea arabica & 76 & 760 & 22,16 & 90 & 6,00 & 0,71 & 2,77 & 10,31 \\
\hline Piptadenia gonoacantha & 5 & 50 & 1,46 & 30 & 2,00 & 4,11 & 16,07 & 6,51 \\
\hline Allophylus edulis & 7 & 70 & 2,04 & 50 & 3,33 & 2,55 & 9,97 & 5,11 \\
\hline Mortas & 21 & 210 & 6,12 & 80 & 5,33 & 0,54 & 2,11 & 4,52 \\
\hline Prunus sellowii & 16 & 160 & 4,66 & 70 & 4,67 & 0,65 & 2,54 & 3,96 \\
\hline Chrysophyllum flexuosum & 15 & 150 & 4,37 & 40 & 2,67 & 1,16 & 4,54 & 3,86 \\
\hline Trichilia lepidota & 13 & 130 & 3,79 & 70 & 4,67 & 0,67 & 2,63 & 3,70 \\
\hline Nectandra lanceolata & 3 & 30 & 0,87 & 30 & 2,00 & 1,91 & 7,48 & 3,45 \\
\hline Citronella megaphylla & 16 & 160 & 4,66 & 40 & 2,67 & 0,34 & 1,34 & 2,89 \\
\hline Anadenanthera peregrina & 11 & 110 & 3,21 & 40 & 2,67 & 0,57 & 2,23 & 2,70 \\
\hline
\end{tabular}


Chrysophyllum flexuosum e Nectandra lanceolata são espécies marcadamente favorecidas por solos de drenagem moderada e com características físicoquímicas semelhantes aos dos sítios aqui estudados (Ferreira Júnior et al. 2007), sendo esta uma possível explicação para seu sucesso nos ambientes amostrados (tabela 2). Assim, espécies como estas aparecem listadas entre as primeiras em IVI provavelmente pelo ambiente proporcionado pelos solos da localidade e, portanto, seu destaque não deve ser atribuído necessariamente ao nível de exposição à trilha. Porém, também se verifica (tabela 2) que, dependendo do nível de influência da trilha, diferentes espécies destacaram-se em maior ou menor grau nos valores dos parâmetros fitossociológicos estimados, podendo este ser um efeito causado pelas alterações abióticas causadas pela trilha.

Em ambos os levantamentos (Mata da Biologia e PEFI), a Categoria das Mortas apresentou valores consideráveis de IVI (tabelas 1 e 2); assim, é interessante que se avalie o quanto as mortas variam entre os diferentes setores. Nesse sentido, como evidencia a tabela 3, a amostragem no PEFI apresentou diferença significativa entre indivíduos mortos em pé nos setores Controle e Fontes do Ipiranga (ANOVA; F $=4,43$; valor-p $<0,05$; Tukey-Kramer: valor- $\mathrm{p}_{\text {Fontes do }}$ Ipiranga/Controle $<0,05$ ), e resultado semelhante ocorreu na Mata da Biologia (tabela 3), na qual houve diferença entre os setores Borda e Controle (ANOVA; $\mathrm{F}=5,13$; valor-p $<0,05$; Tukey-Kramer: valor- $\mathrm{p}_{\text {Borda/Controle }}<$ $0,05)$. A mortalidade decorrente da maior exposição ao vento, a temperaturas mais altas e à umidade menor tem sido relacionada com efeito de borda (Matlack 1994, Rodrigues \& Nascimento 2006), e uma trilha possui, ressalvadas as questões de escala, elementos que a tornam semelhante à borda de um fragmento. Isso ajudaria a explicar as diferenças encontradas nas duas florestas. Porém, o resultado encontrado no PEFI é intrigante, uma vez que o setor de maior impacto potencial (Terra Batida) não diferiu em relação aos demais nesse parâmetro, havendo necessidade de estudos mais aprofundados neste sítio para que a mortalidade ali verificada seja compreendida de modo mais acurado, inclusive considerando possibilidade de processos estocásticos.

Os setores das Fontes do Ipiranga e Terra Batida do PEFI apresentaram variação significativa no parâmetro densidade de indivíduos (ANOVA; F = 4,38; valor-p < 0,05; Tukey-Kramer: valor- $\mathrm{p}_{\text {Fontes do }}$ Ipiranga/Terra Batida $<0,05)$. Para esse parâmetro, na Mata da Biologia, o setor Borda diferiu significativamente em relação a todos os outros três (ANOVA; $F=6,48$;

Tabela 3. Comparação entre as médias de mortas em pé e densidade total de indivíduos das espécies amostradas no Parque Estadual Fontes do Ipiranga, São Paulo, SP, Brasil, e na Mata da Biologia, Viçosa, MG, Brasil. Letras distintas indicam diferença entre as médias e letras iguais indicam médias semelhantes para o teste a posteriori de Tukey-Kramer $(\alpha=0,05)$. Valor-p inferior a 0,05 indica significância estatística (*) para o parâmetro considerado, segundo a ANOVA.

Table 3. Comparison between upright dead plants and total density averages of the species sampling in the Parque Estadual Fontes do Ipiranga, São Paulo, SP, Brazil, and in the Mata da Biologia, Viçosa, MG, Brazil. Different letters indicate statistical differences and same letters indicate that differences between treatments are not significant (Tukey-Kramer post hoc, $\alpha=0,05$ ). P-value $<0,05$ was considered to indicate statistical differences $(*)$ (ANOVA).

Parque Estadual das Fontes do Ipiranga

\begin{tabular}{|c|c|c|c|c|c|}
\hline \multirow[b]{2}{*}{ Parâmetros } & \multicolumn{3}{|c|}{ Setores } & & \multirow[b]{2}{*}{ Valor-p } \\
\hline & Terra Batida & Fontes do Ipiranga & Controle & & \\
\hline Mortas em pé & $1,30( \pm 1,25)^{\mathrm{ab}}$ & $2,70( \pm 1,42)^{\mathrm{a}}$ & $1,00( \pm 1,41)^{\mathrm{b}}$ & & $0,0217 *$ \\
\hline Densidade total & $22,40( \pm 8,28)^{\mathrm{a}}$ & $32,10( \pm 6,94)^{b}$ & $25,70( \pm 7,07)^{\mathrm{ab}}$ & & $0,0225^{*}$ \\
\hline \multicolumn{6}{|c|}{ Mata da Biologia } \\
\hline \multicolumn{6}{|c|}{ Setores } \\
\hline Mortas em pé & $\begin{array}{c}\text { Borda } \\
4,40( \pm 2,41)^{\mathrm{a}}\end{array}$ & $\begin{array}{c}\text { Distância Média } \\
2,00( \pm 1,0)^{\mathrm{ab}}\end{array}$ & $\begin{array}{c}\text { Controle } \\
0,90( \pm 0,88)^{\mathrm{b}}\end{array}$ & $\begin{array}{c}\text { Floresta } \\
2,10( \pm 1,97)^{\mathrm{ab}}\end{array}$ & $0,0064 *$ \\
\hline Densidade total & $50,00( \pm 7,84)^{\mathrm{a}}$ & $36,20( \pm 3,03)^{\mathrm{b}}$ & $33,00( \pm 7,54)^{\mathrm{b}}$ & $34,30( \pm 8.50)^{\mathrm{b}}$ & $0,0020 *$ \\
\hline
\end{tabular}


valor-p $<0,05$; Tukey-Kramer: valor- $\mathrm{p}_{\text {Borda/Dist.Média, }}$ Borda/Controle e Borda/Floresta $<0,05)$. Ambos os resultados podem ser observados na tabela 3. Uma explicação possível seria que a radiação luminosa e temperatura, relativamente elevadas na trilha, poderiam promover maior velocidade de germinação, crescimento, reprodução e defesa contra patógenos e herbívoros, a exemplo das observações realizadas por Denslow (1987) para o comportamento de clareiras. Outro aspecto comum a bordas e que pode se dar nas áreas adjacentes a trilhas refere-se à colonização de espécies de crescimento e reprodução rápida (as pioneiras), o que certamente incrementaria a densidade ali encontrada.

Não houve ligação entre grau de exposição à trilha e proporção de indivíduos anemocóricos, zoocóricos e autocóricos, tanto no PEFI $\left(X^{2}=4,83\right.$, valor-p > 0,05 , tabela 4) como na Mata da Biologia $\left(X^{2}=11,43\right.$, valor-p $>0,05$, tabela 4$)$. Sabe-se que a anemocoria tende a ser favorecida em fisionomias mais abertas (Howe \& Smallwood 1982), nas quais se verifica ausência de um dossel contínuo e, assim, esperavase maior ocorrência dessa síndrome em regiões mais influenciadas pela trilha, o que não foi verificado em nenhum dos fragmentos analisados (PEFI: $X^{2}=2,04$, valor-p > 0,05, tabela 4; Mata da Biologia: $X^{2}=5,60$, valor-p $>0,05$, tabela 4 ). Provavelmente, a trilha e a área de interior de mata amostrada não possuem grau de abertura suficiente a ponto de favorecer indivíduos anemocóricos, podendo estes preferir áreas próximas às bordas da floresta (e não da trilha) ou mesmo grandes

Tabela 4. Valores observados e esperados (entre parênteses) dos indivíduos arbóreos nas síndromes de dispersão, nos setores amostrados no Parque Estadual das Fontes do Ipiranga, São Paulo, SP, Brasil, e na Mata da Biologia, Viçosa, MG, Brasil. São fornecidos também os resultados dos testes qui-quadrado $(\alpha=0,05 \%)$ para cada coluna e para cada linha e o resultado total para cada fragmento florestal.

Table 4. Observed and expected values (in parentheses) of trees along dispersal syndromes in the sectors sampled in the Parque Estadual das Fontes do Ipiranga, São Paulo, SP, Brazil, and in the Mata da Biologia, Viçosa, MG, Brazil. We also supplied the chi-squared results ( $\alpha=0,05 \%)$ for each column and each line, and the total result for each forest.

\begin{tabular}{|c|c|c|c|c|c|c|}
\hline \multicolumn{4}{|c|}{ Parque Estadual Fontes do Ipiranga } & \multirow[b]{3}{*}{ Total } & \multirow[b]{3}{*}{ Qui-quadrado $\left(X^{2}\right)$} & \multirow[b]{3}{*}{ Valor-p } \\
\hline & \multicolumn{3}{|c|}{ Setores } & & & \\
\hline $\begin{array}{l}\text { Síndromes de } \\
\text { dispersão }\end{array}$ & Terra Batida & Ipiranga & Controle & & & \\
\hline Anemocórica & $23(19,26)$ & $3,87)$ & $14(18,87)$ & 62 & 2,0366 & 0,3612 \\
\hline Zoocórica & $223(224,63)$ & $78,29)$ & $225(220,08)$ & 723 & 0,1607 & 0,9228 \\
\hline Autocórica & $1(3,11)$ & ,85) & $3(3,04)$ & 10 & 2,6327 & 0,2681 \\
\hline Total & 247 & 06 & 242 & 795 & 4,8300 & 0,7756 \\
\hline $\begin{array}{l}\text { Qui-quadrado } \\
\left(X^{2}\right)\end{array}$ & 2,1696 & 930 & 1,3674 & & & \\
\hline Valor-p & 0,3380 & 239 & 0,5048 & & & \\
\hline \multirow[t]{4}{*}{ Decisão do teste } & \multicolumn{6}{|c|}{ Não se rejeita $H o$} \\
\hline & \multicolumn{3}{|c|}{ Mata da Biologia } & & & \\
\hline & \multicolumn{3}{|c|}{ Setores } & & & \\
\hline & Borda + Dist. Média & Controle & Floresta & & & \\
\hline Anemocórica & $47(49,17)$ & $34(43,78)$ & $55(43,05)$ & 136 & 5,5977 & 0,0609 \\
\hline Zoocórica & $362(356,49)$ & $\begin{array}{c}324 \\
(317,36)\end{array}$ & $300(312,15)$ & 986 & 0,6970 & 0,7057 \\
\hline Autocórica & $1(4,34)$ & $7(3,86)$ & $4(3,80)$ & 12 & 5,1352 & 0,0767 \\
\hline Total & 410 & 365 & 359 & 1134 & 11,43 & 0,1785 \\
\hline $\begin{array}{l}\text { Qui-quadrado } \\
\left(X^{2}\right)\end{array}$ & 2,7513 & 4,8780 & 3,8006 & & & \\
\hline Valor-p & 0,2527 & 0,0873 & 0,1495 & & & \\
\hline Decisão do teste & & & Não se rejei & & & \\
\hline
\end{tabular}


clareiras, onde a incidência de ventos é certamente maior e, portanto, constituem locais que facilitam a dispersão de propágulos (Nunes et al. 2003).

Por sua vez, a zoocoria predomina amplamente nas florestas tropicais de modo geral (Howe \& Smallwood 1982), com seu tipo principal (endozoocoria) ocorrendo em aproximadamente $75 \%$ das espécies da Mata Atlântica (Almeida Neto et al. 2008). Com esse argumento, aliado ao fato de que a trilha tende a favorecer a passagem de animais, assim como acontece com as estradas (Scoss et al. 2004), seria possível prever que a freqüência de zoocoria não variasse entre os setores. De fato, foi o que se verificou no PEFI $\left(X^{2}=0,16\right.$, valor-p $>0,05$, tabela 4$)$ e na Mata da Biologia $\left(X^{2}=0,70\right.$, valor-p $>0,05$, tabela 4$)$.

De modo geral, os resultados deste trabalho indicaram que as trilhas estudadas acarretam algumas modificações nos trechos de vegetação a elas adjacente. A hipótese do trabalho foi aceita em parte, uma vez que não foi observada diferença na frequência de síndromes de dispersão entre a borda da trilha e o interior de mata, tanto no PEFI como na Mata da Biologia. Porém, são necessários estudos em outras trilhas e em outros fragmentos florestais atlânticos para que se confirmem os padrões aqui detectados. Cabe, também, a sugestão de que futuros estudos de restauração e manejo de comunidades florestais considerem, além do efeito de borda e das clareiras, também o de trilhas como uma variável importante na alteração das características originais dos ecossistemas nativos.

\section{Agradecimentos}

O primeiro autor agradece à CAPES (Coordenação de Aperfeiçoamento de Pessoal de Nível Superior), pela bolsa de Mestrado, e ao Programa de PósGraduação do Instituto de Botânica. Os autores agradecem aos estagiários e demais colaboradores que ajudaram no esforço de campo; aos técnicos dos Herbários SP e VIC; aos especialistas que auxiliaram nas identificações taxonômicas; aos dois revisores anônimos que muito contribuíram para a versão definitiva deste manuscrito; à Juliana Kiomi Rodrigues Hirata, por ter cedido seus dados de campo no PEFI; e, de forma especial, a Walnir Gomes Ferreira Júnior e ao Prof. Alexandre Francisco da Silva (in memoriam).

\section{Literatura citada}

Almeida Neto, M., Campassi, F., Galetti, M., Jordano, P. \& Oliveira Filho, A.T. 2008. Vertebrate dispersal syndromes along the Atlantic Forest: broad-scale patterns and macroecological correlates. Global Ecology and Biogeography 17: 503-513.

Andrade, W.J. 2003. Implantação e manejo de trilhas. In: S. Mitraud (org.). Manual de ecoturismo de base comunitária: ferramentas para um planejamento responsável. WWF-Brasil, Brasília, pp. 247-259.

Antunes, D.F., Silva, M.A.M. \& Alburquerque, A.L.E. 2000. Predação de ovos em um fragmento de floresta atlântica, PE, Brasil. Biota 1: 14-18.

APG II. 2003. An update of the Angiosperm Phylogeny Group classification for the orders and families of flowering plants: APG II. Botanical Journal of the Linnean Society 141: 399-436.

Callegari-Jacques, S.M. 2003. Bioestatística: princípios e aplicações. Artmed, Porto Alegre.

Catharino, E.L.M., Bernacci, L.C., Franco, G.A.D.C., Durigan, G. \& Metzger, J.P. 2006. Aspectos da composição e diversidade do componente arbóreo das florestas da Reserva Florestal do Morro Grande, Cotia, SP. http://www.biotaneotropica.org.br/v6n2/ pt/abstract?article+bn00306022006 (acesso em 01.11.2007).

CIENTEC. 2004. MATA NATIVA 2.0: sistema para análise fitossociológica e elaboração de planos de manejo de florestas nativas. [S.1.:s.n.].

Cole, D.N. 1978. Estimating the susceptibility of wildland vegetation to trailside. The Journal of Applied Ecology 15: 281-286.

Denslow, J.S. 1987. Tropical rainforest gaps and tree species diversity. Annual Review of Ecology and Systematics 18: 431-451.

Dislish, R., Kisser, N. \& Pivello, V.R. 2002. The invasion of a forest fragment in São Paulo (SP) by the Australian palm Archontophoenix cunninghamiana $\mathrm{H}$. Wendl. \& Drude. Revista Brasileira de Botânica 25: 55-64.

Drumm, A. \& Moore, A. 2002. O que é ecoturismo. In: Desenvolvimento do ecoturismo - um manual para os profissionais de conservação, v. 1. The Nature Conservancy, Arlington, pp. 15-22.

Ferreira Júnior, W.G., Silva, A.F., Schaefer, C.E.G.R., Meira-Neto, J.A.A., Dias, A.S., Ignácio, M. \& Medeiros, M.C.M.P. 2007. Influence of soil and topographic gradients on tree species distribution in a Brazilian Atlantic Tropical Semideciduous Forest. Edinburgh Journal of Botany 64: 137-157.

Fidalgo, O. \& Bononi, V.L.R. 1989. Técnicas de coleta, preservação e herborização de material botânico. Instituto de Botânica, São Paulo.

Freixêdas-Vieira, V.M., Passold, A.J. \& Magro, T.C. 2000. Impactos do uso público: um guia de campo para utilização do método VIM. In: Rede Nacional Pró-Unidades de Conservação e Fundação O Boticário de Proteção à Natureza (orgs.). Anais do $2^{\circ}$ Congresso 
de Unidades de Conservação, Campo Grande, v. 2, pp.296-305.

Gomes,E.P.C.\& Mantovani, W. 2001. Size distribution in a warm temperate forest tree populations in São Paulo, Southeastern Brazil. Naturalia 26: 131-158.

Hirata, J.K.R. 2006. Florística e estrutura do componente arbóreo de trilhas do Parque Estadual das Fontes do Ipiranga, São Paulo, SP. Dissertação de Mestrado, Instituto de Botânica, São Paulo.

Howe, H.F. \& Smallwood, J. 1982. Ecology of seed dispersal. Annual Review of Ecology and Systematics 13: 201-228.

Kent, M. 2003. Ecoturism, environmental preservation and conflicts over natural resources. Horizontes Antropológicos 9: 185-203.

Ladeira, A.S., Ribeiro, G.A., Dias, H.C.T., Schaefer, C.E.G.R., Fernandes Filho, E. \& Oliveira Filho, A.T. 2007. O perfil dos visitantes do Parque Estadual do Ibitipoca (PEIb), Lima Duarte, MG. Revista Árvore 31: 1091-1098.

Lopes, W.P., Paula, A., Sevilha, A.C. \& Silva, A.F. 2002. Composição da flora arbórea de um trecho de Floresta Estacional no Jardim Botânico da Universidade Federal de Viçosa (Face Sudoeste), Viçosa, Minas Gerais. Revista Árvore 26: 339-347.

Magurran, A.E. 2004. Measuring biological diversity. Blackwell Publishing, Oxford.

Marangon, L.C., Soares, J.J. \& Feliciano, A.L.P. 2003. Florística arbórea da Mata da Pedreira, município de Viçosa, Minas Gerais. Revista Árvore 27: 207-215.

Martins, F.R. 1991. Estrutura de uma floresta mesófila. Editora da UNICAMP, Campinas.

Matlack, G.R. 1994. Vegetation dynamics of the forest edge: trends in space and sucessional time. Journal of Ecology 82: 113-123.

Meira Neto, J.A.A. \& Martins, F.R. 2002. Composição florística de uma Floresta Estacional Semidecidual Montana no município de Viçosa, MG. Revista Árvore 26: 437-446.

MOBOT. 2007. Missouri Botanical Garden Plant Science. Database - W3 TRÓPICOS. http://www.mobot.org (acesso em 2007).

Mueller-Dombois, D. \& Ellenberg, H. 1974. Aims and methods of vegetation ecology. John Wiley \& Sons, New York.

Nunes, Y.R.F., Mendonça, A.V.R., Botezelli, L., Machado, E.L.M. \& Oliveira Filho, A.T. 2003. Variações da fisionomia, diversidade e composição de guildas da comunidade arbórea em um fragmento de Floresta Semidecidual em Lavras, MG. Acta Botanica Brasilica 17: 213-229.
Oliveira Filho, A.T. \& Fontes, M.A.L. 2000. Patterns of floristic differentiation among Atlantic Forests in southeastern Brazil and the influence of climate. Biotropica 32: 793-810.

Oliveira, R.T., Bloomfield, V.K. \& Magalhães, L.M.S. 1999. Trilhas auto-guiadas: proposta de implantação e interpretação na Floresta Nacional Mário Xavier Sandra Regina da Costa. Floresta e Ambiente 6: 138-143.

Pijl, L.V.D. 1982. Principles of dispersal in higher plants. Springer-Verlag, New York.

Pires, J.S.R., Santos, J.E., \& Pires, A.M.Z.C.R. 2004a. Gestão biorregional. Uma abordagem conceitual para o manejo de paisagens. In: J.E. Santos, F. Cavalheiro, J.S.R. Pires, C.H. Oliveira \& A.M.Z.C.R. Pires (orgs.). Faces da polissemia da paisagem: Ecologia, planejamento e percepção. RIMA, São Carlos, v. 1. pp. 23-34.

Pires, A.M.Z.C.R., Pires, J.S.R. \& Santos, J.E. 2004b. Avaliação da integridade ecológica de bacias hidrográficas. In: J.E. Santos, F. Cavalheiro, J.S.R. Pires, C.H. Oliveira \& A.M.Z.C.R. Pires (orgs.). Faces da polissemia da paisagem: Ecologia, planejamento e percepção. RIMA, São Carlos, v. 1, pp. 123-154.

Robim, M.J., Fonte, M.A. \& Cavalheiro, F. 2004. Determinação da capacidade de carga da Trilha da Praia do Sul no Parque Estadual da Ilha Anchieta: uma aplicação do método de Cinfuentes. In: J.E. Santos, F. Cavalheiro, J.S.R. Pires, C.H. Oliveira \& A.M.Z.C.R. Pires (orgs.). Faces da Polissemia da Paisagem - Ecologia, Planejamento e Percepção. RIMA, São Carlos.

Rodrigues, P.J.F.P. \& Nascimento, M.T. 2006. Fragmentação florestal: breves considerações sobre efeito de borda. Rodriguésia 57: 63-74.

Roncero-Siles, M.F. 2003. Modelagem espacial para atividades de visitação pública em áreas naturais. Dissertação de Mestrado, Instituto de Biociências da Universidade de São Paulo, São Paulo.

Roovers, P., Baeten, S. \& Hermy, M. 2004. Plant species variation across path ecotones in a variety of commom vegetation types. Plant Ecology 170: 107-119.

Scoss, L.M., De Marco Júnior, P., Silva, E. \& Martins, S.V.2004. Uso de parcelas de areia para o monitoramento de impacto de estradas sobre a riqueza de espécies de mamíferos. Revista Árvore 28: 121-127.

Tabarelli, M. \& Mantovani, W. 1999. A riqueza de espécies arbóreas na floresta atlântica de encosta no estado de São Paulo (Brasil). Revista Brasileira de Botânica 22: 217-223.

Veloso, H.P., Rangel Filho, A.L.R. \& Lima, J.C.A. 1991. Classificação da vegetação brasileira, adaptada a um sistema universal. Fundação Instituto Brasileiro de Geografia e Estatística, Rio de Janeiro.

Zar, J.H. 1999. Biostatistical analysis. 4 ed. Prentice-Hall, New Jersey. 